\title{
Adaptive mesh refinement FEM for damage evolution of heterogeneous brittle media
}

\author{
Feng Rong ${ }^{1,4}$, Mengfen Xia ${ }^{1,2}$, Fujiu Ke ${ }^{1,3}$, Yilong Bai ${ }^{1}$ \\ ${ }^{1}$ State Key Laboratory of Nonlinear Mechanics (LNM), Institute of Mechanics, Chinese \\ Academy of Sciences, Beijing 100080, People's Republic of China \\ ${ }^{2}$ Department of Physics, Peking University, Beijing 100871, People's Republic of China \\ ${ }^{3}$ Department of Applied Physics, Beihang University, Beijing, 100088, People's Republic \\ of China \\ E-mail: rongf@lnm.imech.ac.cn
}

Received 17 November 2004, in final form 14 April 2005

Published 1 July 2005

Online at stacks.iop.org/MSMSE/13/771

\begin{abstract}
Damage evolution of heterogeneous brittle media involves a wide range of length scales. The coupling between these length scales is the underlying mechanism of damage evolution and rupture. However, few of previous numerical algorithms consider the effects of the trans-scale coupling effectively. In this paper, an adaptive mesh refinement finite element method (FEM) algorithm is developed to simulate this trans-scale coupling. The adaptive serendipity element is implemented in this algorithm, and several special discontinuous base functions are created to avoid the incompatible displacement between the elements. Both the benchmark and a typical numerical example under quasi-static loading are given to justify the effectiveness of this model. The numerical results reproduce a series of characteristics of damage and rupture in heterogeneous brittle media.
\end{abstract}

\section{Introduction}

Heterogeneous brittle media, such as rock, concrete, etc, are used widely as structural components in infrastructure and engineering. The increasing demands require an adequate damage evolution modelling and the appropriate prediction of overall rupture. Currently, researchers have indicated that damage evolution, especially the rupture of heterogeneous brittle media, has two characteristics, i.e. catastrophe and sample-specificity. It is difficult to predict the catastrophe owing to the sample-specificity.

Previous works have shown that the failure process involves many coupled spatial scales [14-16]. In particular, with increasing load, some disordered details at small scales may

4 Author to whom any correspondence should be addressed. 
be amplified strongly due to strong and sensitive trans-scale coupling and become significant effects on the global rupture resulting in sample-specificity. In order to reveal the characteristics and the underlying mechanism of the damage and rupture, it is necessary to simulate the transscale coupling effect on the whole damage evolution process.

However, generally speaking, few of the previous numerical algorithms consider the effects of the trans-scale coupling on the damage evolution of heterogeneous media effectively. Traditionally, researchers in computational analysis concentrated on bringing more details into their structural system models, but ignored the multi-spatial scales inherent in physical problems. To obtain proper resolution of damage effects, finite element meshes consisting of several million continuum elements are necessary. These often require hours of computation time on even the latest supercomputer and make the analysis of damage evolution almost impossible. This severely limits the usefulness of computation. Therefore, the concept of multiscale algorithm has been induced to solve this dilemma. Many multiscale finite element methods (FEM) have been developed recently. These are, mainly:

(1) $h$-version and $p$-version [3]; in the $h$-version FEM convergence is achieved by mesh refinement; in the $p$-version, it is achieved by increasing polynomial degree.

(2) $h p-d$ version and $s$-version [3,4]; the $h p-d$ version FEM has a combination of $h$ - and $p$-extensions in a hierarchical domain decomposition. The $s$-version FEM accomplishes the idea, which is proposed by Belytschko et al [13], by overlaying arbitrary local mesh on the global mesh.

(3) Generalized FEM [5-7]; the generalized finite element method (GFEM) was first introduced by Melenk [6] and Babuska [7]. It combines the desirable features of the standard FEM and meshless methods. The key difference in the GFEM when compared with the traditional FEM, is the construction of the ansatz space. Each node of the finite element mesh carries a number of ansatz functions expressed in terms of the global coordinate system. These ansatz functions are multiplied by a partition of unity and serve as element ansatz functions in the patch constituted by the elements incident at the node.

The problem concerned in this paper is physically 'multiscale' due to heterogeneities and microdamage. Usually, the multiscale method is dealt with by 'bottom-up' averages, which considered the average effect of grains, micro-cracks or inclusions. This is very effective for a macroscopic average feature, such as stiffness. However, it is well known that in heterogeneous brittle media, the micro-damages are usually initiated on a small scale at some uncertain locations. The micro-damages develop from small to large scales via growth and coalescence and eventually trigger catastrophe. This is a dynamical, trans-scale phenomenon. In order to simulate such phenomena, instead of the usual 'bottom-up' average, we have to turn to an adaptive scheme with a large number of sufficiently fine elements owing to the huge scale-span in the problem. However, the most important point is that, in the usual adaptive algorithm, the constitutive relation almost always remains the same in all meshes (no matter large or small).

In this paper, we present an adaptive mesh refinement FEM model (based on the $h p-d$ version), where only the elements posing danger of damage are to be refined to smaller scales. The intrinsic relation (damage criterion) is a kind of multiscale one in accordance with heterogeneities. First, the governing equations of the model problem, and a finite-element scheme are presented. Second, some critical questions in the model are answered when the serendipity element and the smeared cracked model is adopted in the algorithm. Third, to verify the model, a benchmark problem with a known exact solution is investigated. Finally, we give a numerical example of damage evolution to failure in a heterogeneous brittle medium to justify the effectiveness of this model. It is thus found that the comparison between the adaptive mesh refinement FEM and the whole small-scale simulations (WSFEM) indicates 
that the adaptive mesh refinement FEM can give satisfactory results with sufficient precision and lower cost.

\section{Basic framework}

The key factor in the damage evolution problem is the trans-scale coupling effect. In order to reveal the coupling effects, a new finite element model is proposed to examine this problem.

We start with the general $\mathrm{Hu}$-Washizu variational principle, which deals with three independent fields: displacement $\boldsymbol{u}$, strain $\varepsilon$ and stress $\boldsymbol{\sigma}$ fields. These fields are defined in a domain $V$, whose boundary consists of two parts, $S_{u}$ and $S_{t}$, with prescribed displacements and tractions, respectively. The assumed boundaries are completely arbitrary and mutually independent except for certain restrictions. All governing equations can be replaced by a variational equality:

$\int_{V} \delta \varepsilon^{T} \tilde{\boldsymbol{\sigma}}(\varepsilon) \mathrm{d} V+\delta \int_{V} \boldsymbol{\sigma}^{T}(L \boldsymbol{u}-\boldsymbol{\varepsilon}) \mathrm{d} V=\int_{V} \delta \boldsymbol{u}^{T} \overline{\boldsymbol{b}} \mathrm{d} V+\int_{S_{t}} \delta \boldsymbol{u}^{T} \overline{\boldsymbol{t}} \mathrm{d} S-\delta \int_{S_{u}} \boldsymbol{p}^{T}(\boldsymbol{u}-\overline{\boldsymbol{u}}) \mathrm{d} S$,

which must hold for any admissible variations $\delta \boldsymbol{u}, \delta \varepsilon$ and $\delta \boldsymbol{\sigma}$. In equation (1), the symbol $\delta$ denotes variation, $\tilde{\sigma}(\varepsilon)$ is the stress computed from the assumed strain $\varepsilon$ using the constitutive equations, $L$ the operator transforming displacements into strains, $\bar{b}$ the prescribed body forces and $\overline{\boldsymbol{t}}$ the prescribed tractions (surface forces), $\overline{\boldsymbol{u}}$ the prescribed displacements and $\boldsymbol{p}$ the Lagrangian multiplier defined on the $S_{u}$ in [2].

Variational statement (1), representing the weak form of governing equations, can be exploited when discretizing the problem. We interpolate the unknown fields as

$$
\boldsymbol{u} \simeq \boldsymbol{N} \boldsymbol{u}^{n}
$$

where shape function $N$ is defined on elements of diameter $h$ and maximum polynomial order $p, \boldsymbol{u}^{n}$ collect the degree of freedom corresponding to nodal displacements. Each shape function (corresponding either to a displacement mode in the $x$ - or in the $y$-direction in twodimensional problem) is usually formed from a scalar shape function $N^{n}$ by $N^{2 n-1}=\left\{N^{n}, 0\right\}^{T}$ and $N^{2 n}=\left\{0, N^{n}\right\}^{T}$.

Substituting the approximation, equation (2), into the variational equality (1), we obtain a linear equation system for the unknown node displacements $\boldsymbol{u}^{n}$,

$$
\delta\left(\boldsymbol{u}^{n}\right)^{T} \int_{V}(L \boldsymbol{N})^{T} \tilde{\sigma}\left(L \boldsymbol{N} \boldsymbol{u}^{n}\right) \mathrm{d} V=\delta\left(\boldsymbol{u}^{n}\right)^{T}\left(\int_{V} \boldsymbol{N}^{T} \overline{\boldsymbol{b}} \mathrm{d} V+\int_{S_{t}} \boldsymbol{N}^{T} \overline{\boldsymbol{t}} \mathrm{d} S\right) .
$$

In addition, for a given state the constitutive relation of the linear problem is described by

$$
\tilde{\boldsymbol{\sigma}}(\varepsilon)=\boldsymbol{\sigma}=\boldsymbol{D}_{R} \varepsilon-\boldsymbol{D} \varepsilon_{0},
$$

with the prestrain vector $\varepsilon_{0}$. The linear elasticity matrix $\boldsymbol{D}_{R}$ and $\boldsymbol{D}$ depend on the assumption of the problem, such as plane stress or plane strain conditions in two-dimensional problems. Substituting (4) into (3) and taking into account the independence of variations, we obtain the discretized equation

$$
\boldsymbol{K} \boldsymbol{u}^{n}=\boldsymbol{f},
$$

where the stiffness matrix is

$$
\boldsymbol{K}=\int_{V}(L \boldsymbol{N})^{T} \boldsymbol{D}_{R}(L \boldsymbol{N}) \mathrm{d} V
$$




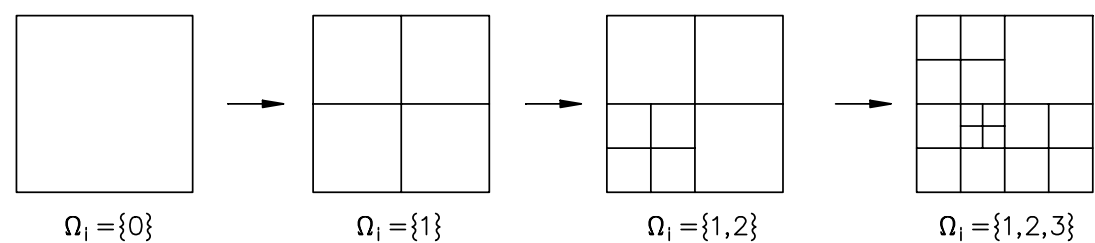

Figure 1. The grids hierarchy.

and the load vector

$$
\boldsymbol{f}=\int_{V} \boldsymbol{N}^{T} \overline{\boldsymbol{b}} \mathrm{d} V+\int_{S_{t}} \boldsymbol{N}^{T} \overline{\boldsymbol{t}} \mathrm{d} S+\int_{V}(L \boldsymbol{N})^{T} \boldsymbol{D} \varepsilon_{0} \mathrm{~d} V .
$$

The interpolations of stress and strain can be discontinuous, hence we can select the interpolation functions such that each stress or strain parameter is associated with only one finite element. The global equations contain the standard displacement degrees of freedom, $\boldsymbol{u}^{n}$ only. Therefore, equation (5) written for one finite element occupies a certain volume $V_{\mathrm{e}}$. Meanwhile, the left vector (external force vector) is then replaced by the contribution of the current element to the internal forces.

\section{Adaptive mesh refinement FEM}

In the previous section, the standard FEM has been introduced. The concept of the adaptive mesh refinement finite element model (AMRFEM) is outlined in this section. The AMRFEM is based on the $h p-d$ version FEM, i.e. the degree of polynomial interpolation functions is fixed, while the mesh is refined as damage occurs in the evolution.

\subsection{Hierarchy of grids}

There are many multiscale numerical models, which adopt the sequence of cell averages based on a finite element discretization. It will make the model easily applicable to trans-scale problems. However, this convenience is based on the local mean field theory, which may discard some significant trans-scale effects on catastrophic failure. In order to delineate the trans-scale effects and have different scales solved together, we introduce a sequence of grids $\Omega_{i}=\{0, \ldots, i\}, i=0, \ldots, I$. The coarsest grid is indicated by $i=1$, while the finest scale we consider is indicated by $i=I$ ( $i=0$ stands for the whole structure). A simple example is shown in figure 1 , where a coarse grid is successively refined with increasing refinement level. This sequence is called a grids hierarchy. Note that the framework presented here is not restricted to this simple configuration, but can also be applied to unstructured and irregular grid refinement if we introduce one mapping function which can map the irregular grid to the structured grid. In addition, we assume without lost of generality, that the computational domain and hence, the grids hierarchy, is unchanged in the paper.

The key factor in the grids hierarchy is the adaptation of shape base function which can guarantee both the continuity of structure and the connectivity of different scales. In finite element analysis, interpolation theories based on Lagrangian and Hermitian interpolation polynomials have produced a variety of useful finite elements. Lagrangian rectangular elements are conceptually simple, but are of limited use due to the large number of internal nodes. Nevertheless, they provide a natural introduction to interpolation functions of serendipity elements which have no internal nodes. The serendipity rectangular element (shown in figure 2) 


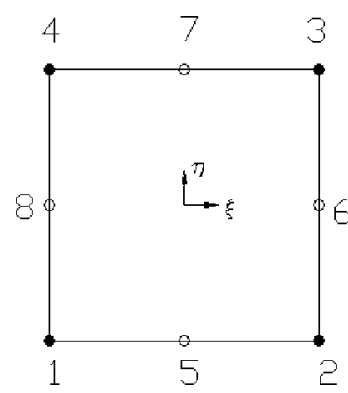

Figure 2. The 4-8 variable nodes serendipity element

is applied to our problem. The discontinuous polynomial interpolation functions which satisfy the $C_{0}$ continuity are used in the model to connect different scales with each other, smoothly.

In addition, based on the variational principle, the stiffness matrix of the whole problem can be set up and solved in one equation.

\subsection{Self-adaptive mesh topology and material heterogeneity}

The self-adaptive mesh topology is adopted in the model. All elements have multi-levels. Computation starts with the coarsest grid. However, with increasing load, some larger scale elements become dangerous to damage; then, these dangerous elements will be adaptively refined to smaller scale grids in the local area. As an element is refined to the smallest scale, it will keep intact or, be broken according to a particular criterion. The criterion is expressed by the comparison between the threshold of the element and the current stress on the element. In this paper, we consider the media with frozen heterogeneity, which means that the threshold of the finest elements is pre-defined randomly and follows a given probability distribution function. The stress on each element is determined by damage-induced stress redistribution, which is a non-linear dynamical process. Therefore, the self-adaptive refined procedure is governed by the coupling between the damage-induced dynamical nonlinearity and the random heterogeneity on multi-scales.

For example, the Coulomb criterion [1]:

$$
F=|\tau|+\mu \sigma-S_{0} \geqslant 0,
$$

can be used. In equation (6), coefficient of friction $\mu$, and inherent shear strength $\tau_{S}$ are two parameters of the Coulomb criterion. The physical meaning of this inequality is as follows. Firstly, if the stress state of an element does satisfy this inequality, it will be adaptively refined to four smaller elements; otherwise the mesh topology will not be modified. Secondly, provided the smaller element still satisfies the inequality, this adaptive refining will continue till the finest element. Finally, when the finest element satisfies this inequality, it will be set damaged.

\subsection{Constitutive relation}

The FEM is based on a constitutive relation. The elastic-brittle constitutive relation is adopted in our model. When the sample is loaded in the compressive load, the response of damaged element will alternate due to the stress situation, i.e. the stiffness of the element will vary according to the difference of force on the damage direction. Actually, the smeared crack model has been widely used for concrete and rocks in [9], but needs modifying for its own feedback. The other relevant model is the rotating crack model based on the evaluation of the 


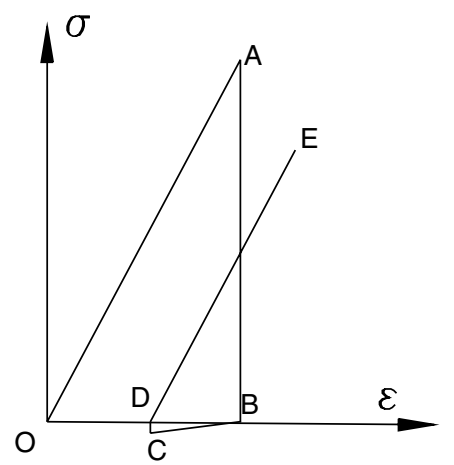

Figure 3. The schematic example of constitutive relation.
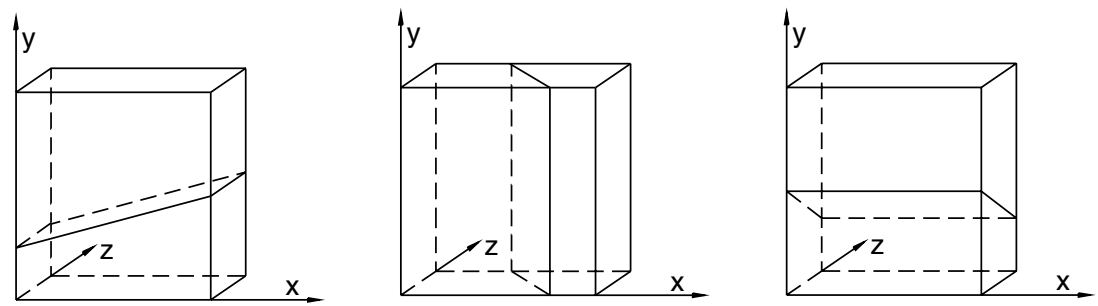

Figure 4. Three-dimensional damage in elements.

damaged material stiffness matrix from the nonlocal average of the total strain in [10]. So, our modified smeared crack model is based on the rotating crack model. That is to say, after an element has become damaged and the force on the direction of damage is the tension, the behaviour of the failed element can be described through a reduced Young's modulus. On the other hand, if the force is the compression, Young's modulus will recover to simulate crack closing. In the meantime, the strain of a damaged element will hold due to the impossibility of the strain recovery at damage. Figure 3 gives a schematic of the constitutive relation.

In the diagram, the response of an intact element will follow line OA, and when the stress reaches $\sigma_{A}$, brittle damage will occur on the orientation of stress, which can be determined with the crack initiation criterion (equation (6)). The element will retain the strain $\varepsilon_{A}$ and sustain further loading. In figure 3, the assuming nominal stress on the crack direction is the tension. So the Young's modulus will be reduced to reflect the effect of the damage. When stress reaches $\sigma_{C}$, the nominal stress changes to compression and the damaged element will switch to a state similar to the intact one. Then Young's modulus will switch to the undamaged state. For simplicity, the stiffness matrix $\boldsymbol{D}_{R}$ and $\boldsymbol{D}$ are assumed to be identical.

The multiple damage orientations are allowed in the simulation. Since three-dimensional crack is allowed in the simulation, damage will cause the relevant modulus to reduce in that axis. The damage of an element may appear in three directions, so that the elements can be distinguished as intact, partially damaged or entirely damaged (figure 4).

\subsection{The basic procedure of AMRFEM}

Figure 5 shows the flow chart of the AMRFEM Algorithm. 


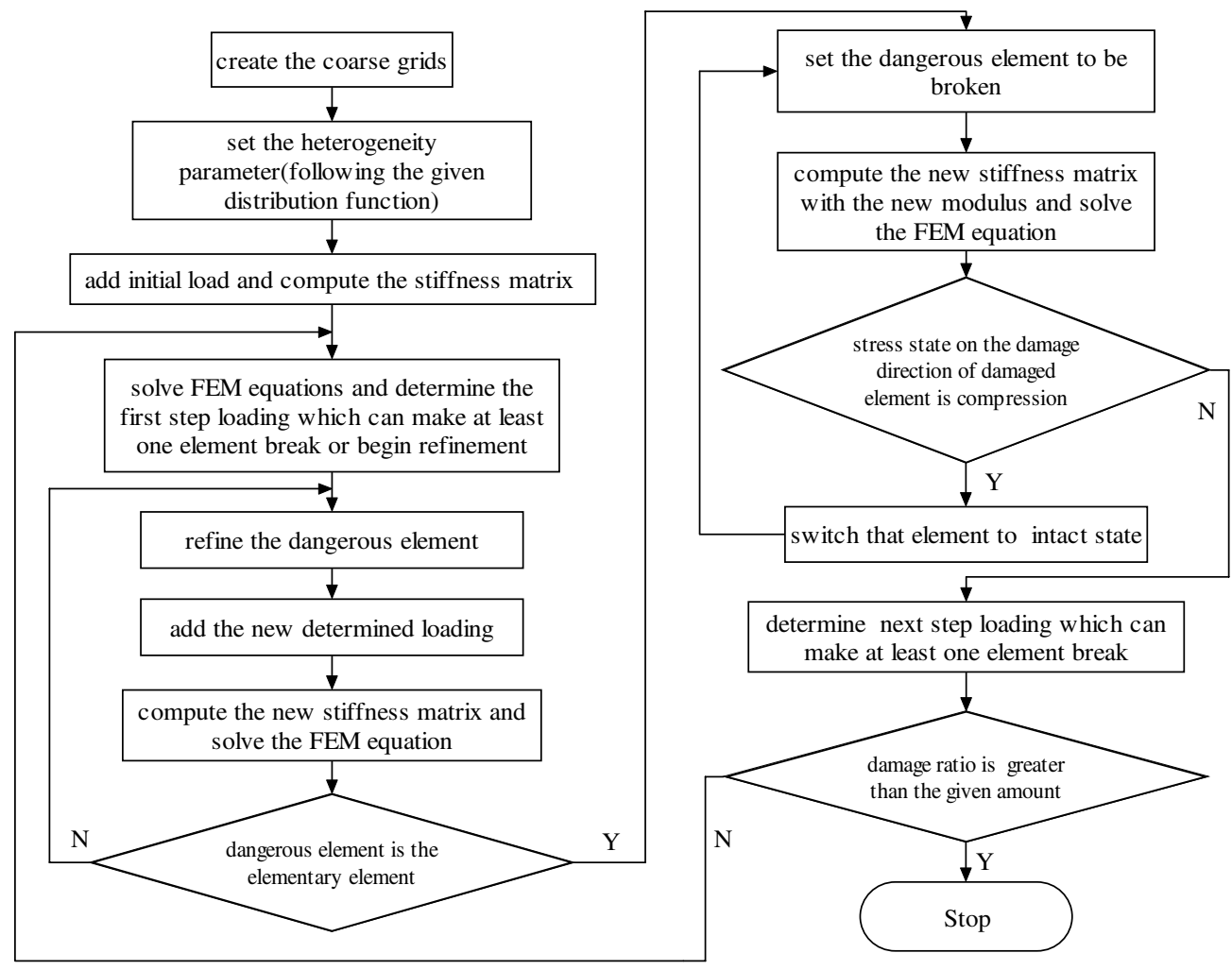

Figure 5. Flow chart of AMRFEM.

\section{Benchmark}

The key points of AMRFEM have been given in the previous section. To verify the effectiveness of the algorithm, a benchmark problem with a known exact solution is investigated. As a typical two-dimensional problem with singularities, the L-shaped domain in plane strain condition shown in figure 6 is considered. The convergence of the AMRFEM procedure can be studied and the accuracy of various FEM versions can be investigated. The boundary conditions are prescribed so that the analytic solution can be computed and corresponds to a MODE 1 loading of the structure [11]. At the re-entrant corner the exact displacement solution can be expressed by an asymptotic expansion, i.e.

$$
\begin{aligned}
& u_{x 1}=\frac{A_{1}}{2 G} r^{\lambda_{1}}\left[\left(3-4 v-Q_{1}\left(\lambda_{1}+1\right)\right) \cos \left(\lambda_{1} \alpha\right)-\lambda_{1} \cos \left(\lambda_{1}-2\right) \alpha\right], \\
& u_{y 1}=\frac{A_{1}}{2 G} r^{\lambda_{1}}\left[\left(3-4 v+Q_{1}\left(\lambda_{1}+1\right)\right) \sin \left(\lambda_{1} \alpha\right)+\lambda_{1} \sin \left(\lambda_{1}-2\right) \alpha\right],
\end{aligned}
$$

which can be written in the form

$$
\overrightarrow{\boldsymbol{u}}_{1}=\frac{A_{1}}{2 G} r^{\lambda_{1}}\left\{\Psi_{1}(\theta)\right\}
$$

In (7) and (8), $G$ is the modulus of rigidity, and $v$ is the Poisson's ratio. Here polar coordinates $r$ and $\alpha$ are centred at the re-entrant corner and the exponent $\lambda_{1}$ characterizes the smoothness of the solution. The coefficient $A_{1}$ is called generalized stress intensity factor and 


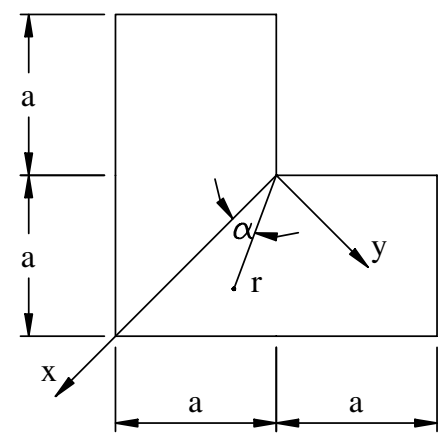

Figure 6. L-shaped plane elastic body.
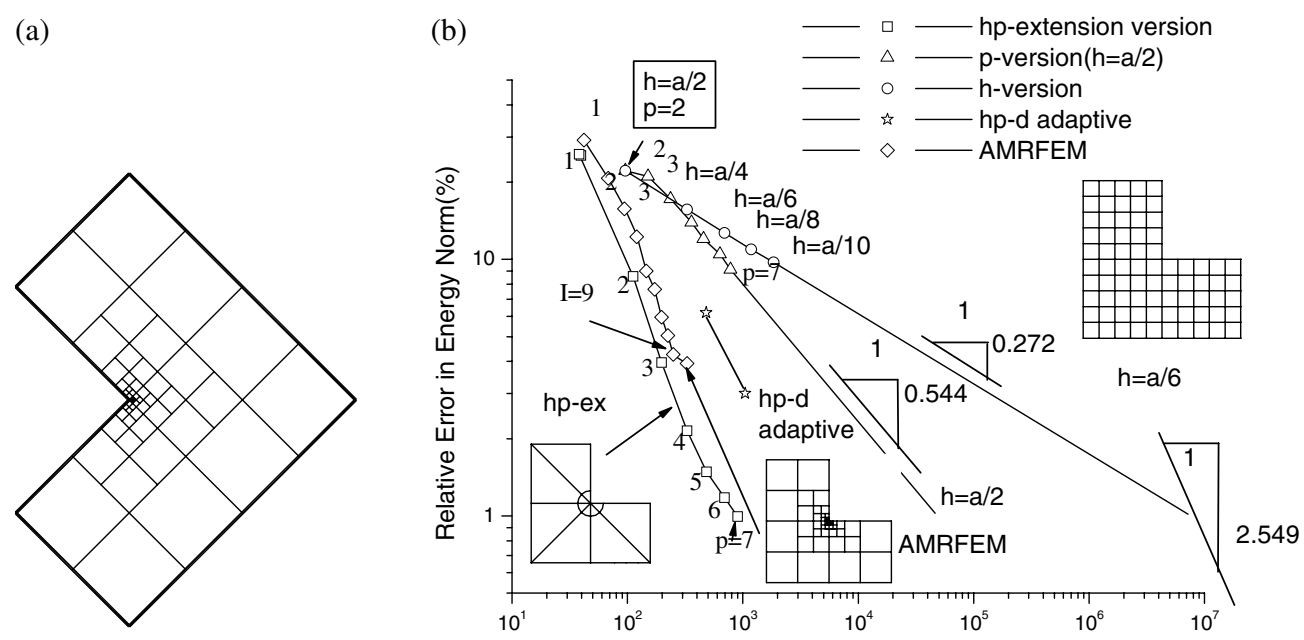

Figure 7. L-shaped AMRFEM grids hierarchy $(a)$ and convergence of AMRFEM (compared with the methods in $[4,11])(b)$.

$Q_{1}$ can be calculated through $\lambda_{1}$. The exact solutions were discussed in detail in [11, p $\left.173 \mathrm{ff}\right]$, where $\lambda_{1}=0.544483737$ and $Q_{1}=0.543075579$ are obtained. In this case, the numerical values of the constants are assumed: Poisson's ratio $v$ is 0.3 , Young's modulus $E$ and the thickness of problem $t$ are set to $1.0, a=1.0$ and the stress intensity factor is closely related to that in $[12,13]$. The strain energy of the exact solution is computed as $\|u\|_{E}^{2}=4.15454423$.

To study the convergence of AMRFEM, nine different-scale grids are generated (see figure 7(a)). The L-shaped domain is loaded with the tractions from the MODE 1 stress tensor. In addition, the edges of re-entrant corner are the free edges, the other edges being clamped with symmetry conditions. To eliminate the rigid body motion, the displacement of the node at the re-entrant corner is set to zero. The relative error of the AMRFEM solution is expressed through the difference between energy norm of exact solution and the AMRFEM solution of

$$
\eta=\frac{\|u\|_{\mathrm{E}}^{\mathrm{FE}}-\|u\|_{\mathrm{E}}^{\mathrm{exa}}}{\|u\|_{\mathrm{E}}^{\mathrm{FE}}},
$$

where $\|u\|_{\mathrm{E}}^{\mathrm{FE}}$ is the energy norm of AMRFEM solution and $\|u\|_{\mathrm{E}}^{\text {exa }}$ is the energy norm of exact solution. 
Table 1. The percentage of relative error in energy norm.

\begin{tabular}{llllll}
\hline$I=1$ & $I=2$ & $I=3$ & $I=4$ & $I=5$ & $I=6$ \\
$\mathrm{DOF}=42$ & $\mathrm{DOF}=68$ & $\mathrm{DOF}=94$ & $\mathrm{DOF}=120$ & $\mathrm{DOF}=146$ & $\mathrm{DOF}=172$ \\
\hline $29.1675 \%$ & $20.7246 \%$ & $15.9564 \%$ & $12.4565 \%$ & $9.0201 \%$ & $7.6370 \%$ \\
$I=7$ & $I=8$ & $I=9$ & $I=10$ & $I=11$ & $I=12$ \\
$\mathrm{DOF}=198$ & $\mathrm{DOF}=224$ & $\mathrm{DOF}=250$ & $\mathrm{DOF}=276$ & $\mathrm{DOF}=302$ & $\mathrm{DOF}=328$ \\
\hline $5.9475 \%$ & $5.0406 \%$ & $4.2613 \%$ & $4.1136 \%$ & $4.0164 \%$ & $3.9274 \%$ \\
\hline
\end{tabular}

Table 1 gives the convergence of the discretization. In particular, the relative error is less than 5\% (4.2613\%) when the grids hierarchy reaches 9 . And we now compare it to the well-known $h$-, $p$-, and $h p$-extensions and $h p$ - $d$ adaptive of the FEMs. In figure $7(b)$, the effect of increasing $I$ can be studied on the log-log graph. As in [11], the typical curves of an $h p$-extension on a geometrically refined mesh and the convergence of an adaptive $h$-version and $p$-version are given. The relative errors in the energy norm of the $p$-version, $h p$-extension descend very fast at the beginning of the DOF increase since the key point of the error is still in the smooth part of the solution. Therefore, the high convergence of the $p$-version for smooth solutions is observed. When the error at the corner becomes dominant, the convergence rate slows down to $\lambda_{1}$. The singularity of the corner influences the discretization with the adaptive $h$-version mesh from the beginning of the refinement process. The dot curve (standing for the $h$-version) is therefore flatter than the $p$-version FEM. Moreover, we can see that the convergence of AMRFEM is very close to the $h p$-extension version FEM with its exponential interpolation function at the beginning of the refinement process (the steepest rate is about 2.549). Finally, the convergence rate slows down because the method is based potentially on the $h p$ - $d$ version FEM. Based on [4], the $h p$ - $d$ adaptive FEM at fixed polynomial $p=3$ is also given in figure $7(b)$. Although the error is smaller than AMRFEM, the DOF consumption of the $h p$ - $d$ adaptive FEM is relatively greater than AMRFEM.

In short, the AMRFEM with grids hierarchy is superior to nomal $h$-extensions and to the $p$-extension on a uniform mesh. The DOF consumption of AMRFEM is smaller than the $h p$ - $d$ adaptive FEM with the same error in energy norm. Nevertheless, its efficiency is still slightly less than that of a $p$-extension on a geometric mesh with its exponential preasymptotic range of convergence. However, for heterogeneous media, due to the hierarchy of grids and the high-efficiency convergence, the AMRFEM is naturally suited to the relevant trans-scale problems. A numerical example of catastrophic failure in a heterogeneous medium will be given in the following section.

The previous benchmark can only give us the convergence of this method. However, due to the impossibility of obtaining the exact stress redistribution after the damage evolution, the previous benchmark can only be used before any damage occurs in the domain.

\section{Numerical example}

In order to illustrate the validity of the proposed model, a numerical example of catastrophic rupture in a heterogeneous brittle medium is given. In the example (figure 8), the failure criterion of the mesoscopic element is of the Coulomb criterion (equation (7)). Obviously, each mesoscopic element has two parameters for failure, the coefficient of friction $\mu$, and inherent shear strength $\tau_{\mathrm{S}} . \mu$ is assumed to be the same for all elements, but $\tau_{\mathrm{S}}$ is different and follows the Weibull distribution in [8], which is used to describe the material heterogeneity, $h\left(\tau_{\mathrm{S}}\right)=m\left(\tau_{\mathrm{S}}^{m-1} / \eta^{m}\right) \exp \left[-\left(\tau_{\mathrm{S}} \eta\right)^{m}\right]$, where $\eta, m$ are two parameters in the Weibull distribution 


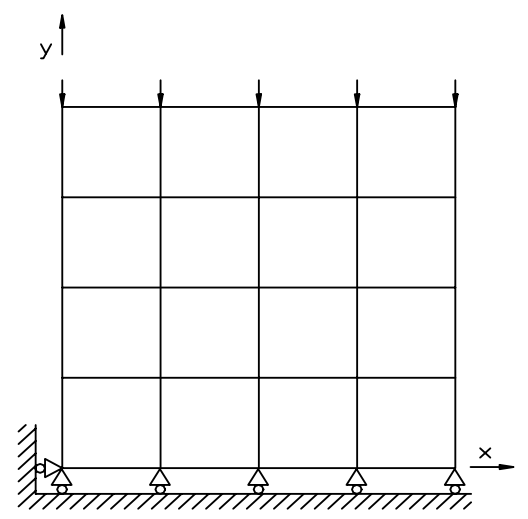

Figure 8. The topology of numerical example.

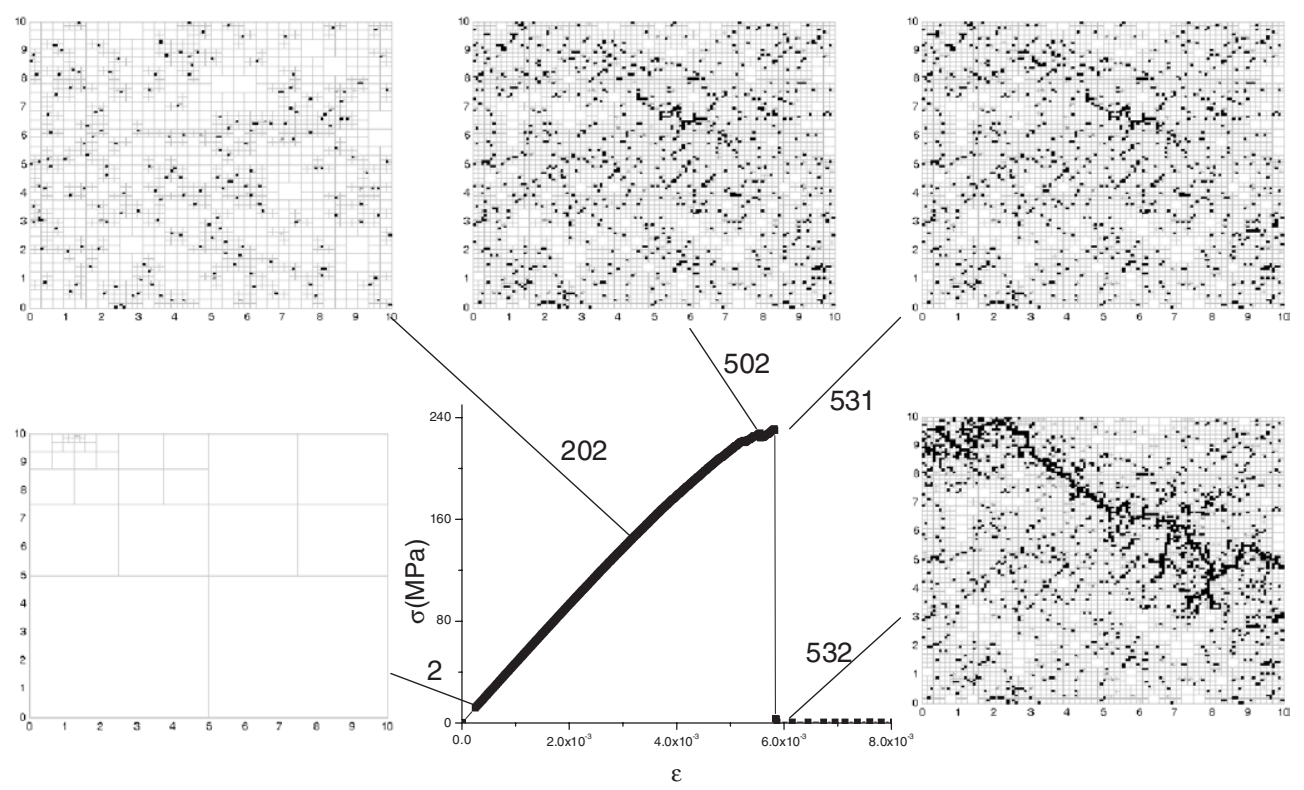

Figure 9. The damage pattern evolution. The number on the arrow line is the quasi-static loading step number. In the pattern, there are three kinds of darkness; black indicates that the elements have been entirely failed; while gray indicates that the elements have been partially failed and white means that the elements are intact.

which stand for the scale parameter and Weibull modulus (shape parameter), respectively. In the example, $\mu=0.639, m=3$ and $\eta=3.10 \times 10^{5} \mathrm{~Pa}$ as given in [1] and [17] for marble. All elements are linear elastic at the beginning, but the modulus will reduce to $1 / R\left(R=10^{5}\right.$ in the case study) of the initial, when the element fails. Although the example is a plane stress problem, the damage is allowed to be a three-dimensional one, which is shown in figure 4. A damage evolution pattern obtained with the AMRFEM algorithm is given in figure 9. It clearly shows the transition from random accumulation of damages to catastrophic rupture owing to coalescence of damage. In addition, we compare this result with that of the simulation with elements of the same finest size, see figure 10. 

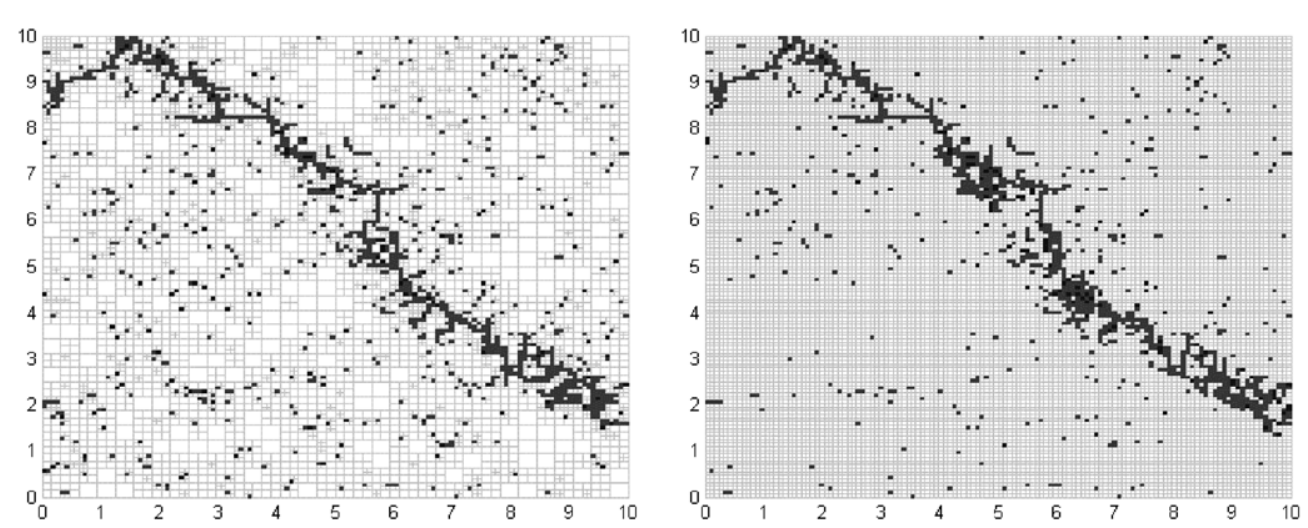

Figure 10. The damage pattern of two simulations, left is the multiscale simulation and right is the whole small-scale simulation.

In order to validate the effectiveness of the proposed AMRFEM algorithm in dealing with the failure in heterogeneous media, we define an equivalent damage ratio $D_{j}=$ $\sum_{i=1}^{\text {elenum }} A_{i}^{(j)} / N$, where $A_{i}^{(j)}=\{0,0.5,1.0\}$ (the partially failed element is defined as 0.5 ) and $N$ is the total of elements. The damage ratio of the state after catastrophe is $7.15 \%$ for AMRFEM, while it is $7.37 \%$ for WSFEM. It can be seen that the precision of the AMRFEM result is good enough if the simulation with WSFEM is regarded as the standard. More importantly, the computational time of the two simulations are $42.6 \mathrm{~h}$ and $64.4 \mathrm{~h}$, respectively. Obviously, the AMRFEM algorithm is much more economical in time consumed for computation, which is extremely significant in large-scale computations.

However, as shown in figure 9, the catastrophic rupture cannot be predicted only from the $\sigma-\varepsilon$ curve. Actually, from our computations, we found that the stress redistribution resulting from the damage interaction plays a critical role in the catastrophic rupture, which we will report in another paper [18] with more computations to clarify the significant mechanism governing the trans-scale phenomenon.

\section{Conclusion}

In order to handle the trans-scale coupling between the damage of mesoscopic scale and the heterogeneity in the damage evolution and rupture in heterogeneous brittle media, a selfadaptive mesh refinement finite element model (AMRFEM) is proposed. The serendipity element is applied to connect the scales at different levels, while the smeared crack model is implemented to avoid the moving inner boundary in damage evolution.

Various numerical examples show definitely, the effectiveness of this AMRFEM modelling simulation of trans-scale problems. First, both benchmark and simulations of damage evolution to failure in heterogeneous medium demonstrate that the AMRFEM results can give a very good precision. Second, the AMRFEM algorithm is much more economical in the time consumed in computation, which is extremely significant in large-scale, trans-scale computations. Finally, the simulations show clearly, the transition from random damage accumulation to catastrophic failure, owing to the coalescence of damage. This indicates that the AMRFEM is a very helpful tool in coping with trans-scale phenomena, which can ensure both, the effectiveness and the low-cost of computation. Therefore, AMRFEM is beneficial not only in revealing the characteristics and underlying mechanism of catastrophe phenomenon, but also in seeking a clue to predicting the catastrophic rupture. 


\section{Acknowledgments}

This work is supported by the National Natural Science Foundation of China (Grant No 10172084, 10472118, 10232050, 10302029 and 10372012), the Major State Research Project 'Nonlinear Science' G200007735 and the Chinese Academy of Sciences.

\section{References}

[1] Jarger J C and Cook N G W 1979 Fundamentals of Rock Mechanics 3rd edn (London: Chapman and Hall)

[2] Zienkiewicz O C and Taylor R L 1988 The Finite Element Method 4th edn, vol 1 (New York: McGraw-Hill)

[3] Fish J 1992 Comput. Struct. 43 539-47

[4] Krause R and Rank E 2003 Comput. Methods Appl. Mech. Eng. 192 3959-83

[5] Stouboulis T, Copps K and Babuška I 2001 Comput. Methods Appl. Mech. Eng. 190 4081-193

[6] Melenk J M 1995 PhD Dissertation University of Maryland, College Park, MD

[7] Babuska I and Melenk J M 1996 Comput. Methods Appl. Mech. Eng. 139 289-314

[8] Weibull W 1951 ASME Trans. J. Appl. Mech. 18 293-7

[9] Bažant Z P and Jirásek M 2002 J. Eng. Mech. 128 1119-49

[10] Jirásek M and Zimmermann T 1998 J. Eng. Mech. 124 277-84

[11] Szabo B A and Babuška I 1991 Finite Element Analysis (New York: Wiley)

[12] Szabo B A, Yosibash Z 1995 Int. J. Numer. Methods Eng. 38 2055-82

[13] Szabo B A and Yosibash Z 1996 Int. J. Numer. Methods Eng. 39 409-34

[14] Bai Y L, Xia M F, Ke F J and Li H L 1998 Rheology of Bodies with Defects ed R Wang (Dordrecht: Kluwer) pp 55-66

[15] Xia M F, Wei Y J, Ke F J and Bai Y L 2002 Pure Appl. Geophys. 159 2491-509

[16] Zhang X H, Rong F, Jia Z K, Ke F J, Xia M F and Bai Y L 2004 Theor. Appl. Fract. Mech. 41 381-9

[17] Xu X H, Ma S P, Xia M F, Ke F J and Bai Y L 2004 Theor. Appl. Fract. Mech. 42 131-8

[18] Rong F, Wang H Y, Xia M F, Ke F J and Bai Y L 2005 Int. J. for Multiscale Comput. Eng. accepted 